\title{
Breathing pattern and chest wall volumes during exercise in patients with cystic fibrosis, pulmonary fibrosis and COPD before and after lung transplantation
}

\author{
H Wilkens, ${ }^{1}$ B Weingard, ${ }^{1}$ A Lo Mauro, ${ }^{2}$ E Schena, ${ }^{2}$ A Pedotti, ${ }^{2}$ G W Sybrecht, ${ }^{1}$ \\ A $_{\text {Aliverti }}^{2}$
}

- Supplementary tables are published online only. To view these files please visit the journal online (http://thorax.bmj. com)

${ }^{1}$ Innere Medizin V, Universitätsklinikum des Saarlandes, Homburg/Saar, Germany

${ }^{2}$ Dipartimento di Bioingegneria, Politecnico di Milano, Italy

\section{Correspondence to}

Andrea Aliverti, Dipartimento di Bioingegneria, Politecnico di Milano, P.zza L. da Vinci, 32. 20133 Milano, Italy: andrea.aliverti@polimi.it

Received 25 November 2009 Accepted 25 May 2010

\begin{abstract}
Background Pulmonary fibrosis (PF), cystic fibrosis (CF) and chronic obstructive pulmonary disease (COPD) often cause chronic respiratory failure (CRF).

Methods In order to investigate if there are different patterns of adaptation of the ventilatory pump in CRF, in three groups of lung transplant candidates with $\mathrm{PF}(\mathrm{n}=9$, forced expiratory volume in $1 \mathrm{~s}\left(\mathrm{FEV}_{1}\right)=37 \pm 3 \%$ predicted, forced vital capacity $(\mathrm{FVC})=32 \pm 2 \%$ predicted), $\mathrm{CF}\left(\mathrm{n}=9, \mathrm{FEV}_{1}=22 \pm 3 \%\right.$ predicted, $\mathrm{FVC}=30 \pm 3 \%$ predicted) and COPD ( $\mathrm{n}=21$, $\mathrm{FEV}_{1}=21 \pm 1 \%$ predicted, $\mathrm{FVC}=46 \pm 2 \%$ predicted), 10 healthy controls and 16 transplanted patients, total and compartmental chest wall volumes were measured by opto-electronic plethysmography during rest and exercise.
\end{abstract}

Results Three different breathing patterns were found during CRF in PF, CF and COPD. Patients with COPD were characterised by a reduced duty cycle at rest and maximal exercise $(34 \pm 1 \%, p<0.001)$, while patients with PF and CF showed an increased breathing frequency (49 \pm 6 and $34 \pm 2 / \mathrm{min}$, respectively) and decreased tidal volume $(0.75 \pm 0.10$ and $0.79 \pm 0.07$ litres) $(p<0.05)$. During exercise, end-expiratory chest wall and rib cage volumes increased significantly in patients with COPD and CF but not in those with PF. End-inspiratory volumes did not increase in CF and PF. The breathing pattern of transplanted patients was similar to that of healthy controls

Conclusions There are three distinct patterns of CRF in patients with PF, CF and COPD adopted by the ventilatory pump to cope with the underlying lung disease that may explain why patients with $\mathrm{PF}$ and $\mathrm{CF}$ are prone to respiratory failure earlier than patients with COPD. After lung transplantation the chronic adaptations of the ventilatory pattern to advanced lung diseases are reversible and indicate that the main contributing factor is the lung itself rather than systemic effects of the disease.

\section{INTRODUCTION}

The indications for lung transplantation have spanned the spectrum of lung diseases, with chronic obstructive pulmonary disease (COPD), idiopathic pulmonary fibrosis (PF) and cystic fibrosis (CF) being the three most frequent diseases in which this procedure is performed. The distribution primarily reflects the natural history of these lung diseases, with progressive chronic respiratory failure ultimately leading to death unless a transplantation is possible. Current criteria for lung transplantation include a clinically and physiologically severe disease with ineffective or unavailable medical treatment and limited life expectancy of $<2-3$ years. ${ }^{1} 2$ Since there are marked disease-specific differences, with a much lower survival rate for patients with PF than for patients with CF or COPD, there are diseasespecific guidelines for referral. ${ }^{2-4}$ In individual patients it is difficult to predict the clinical course. Given a similar impaired pulmonary function, some patients stay stable for long periods, while others develop respiratory failure within a short period.

Altered patterns of breathing during rest and exercise are observed in these lung diseases. ${ }^{5}$ In their classical studies, Otis, Fenn and Rahn indicated how minimal work of breathing is determined by the different types of load imposed on the ventilatory pump, namely elastic and resistive. ${ }^{6}$ When, like in PF, the elastic load increases, minimal work of breathing would require a rapid shallow breathing pattern. When, like in COPD, the lungs are hypercompliant and the airways obstructed, minimal work would entail a slow deep breathing pattern. When, like in CF, both resistive and elastic load are increased, it could be difficult to predict what the breathing pattern should be to minimise the work of breathing.

The major question of the present study was whether in chronic respiratory failure due to PF, CF and COPD there are different ways of coping with the disease resulting in different patterns of adaptation of the ventilatory pump in very advanced disease state. Therefore, we tested the hypothesis that the different characteristics of these diseases lead to different breathing patterns at rest and during exercise and that chronic adaptations of the ventilatory pattern are reversible after lung transplantation.

\section{METHODS \\ Subjects}

Thirty-nine patients on the waiting list for lung transplantation in the university clinic of Homburg with severe CF $(n=9), P F(n=9)$ and COPD $(n=21)$ diagnosed by accepted criteria were recruited into the study. All patients with COPD, four patients with PF and none of the patients with CF had been former smokers and had abstained from smoking for at least 6 months.

In addition, we studied 16 transplanted patients (T), 5 with COPD (T-COPD), 4 with CF (T-CF) and 7 with PF (T-PF) in a stable condition at least 3 months after lung transplantation. Of these, 7 
patients (4 T-CF and $3 \mathrm{~T}$-COPD) had received double lung and 9 (2 T-COPD, 7 T-PF) single lung transplantation. Analysis of their pulmonary function data before transplantation split into the three diseases did not show any significant difference from the corresponding groups (see online data repository).

All patients were studied during an ongoing continuous pulmonary rehabilitation programme before lung transplantation and were familiar with physiological exercise testing. Components of the pulmonary rehabilitation programme included comprehensive evaluation of medical and nutritional needs, setting of goals for education and exercise training, exercise training (endurance, lower extremity, flexibility, strengthening and upper extremity), education about medical treatments and nutritional counselling.

Ten non-smoking volunteers recruited from hospital staff were considered as the healthy control (HC) group. They followed the same measurement protocol except for 6 min walk and blood gas analysis. Characteristics of both patients and controls are reported in table 1.

The protocol was approved by the local research ethics committee and informed consent was obtained from all patients.

\section{Study design}

Initially, patients were assessed for pulmonary function. Spirometry, lung transfer factor for carbon monoxide and subdivisions of lung volumes by body plethysmography (Masterlab; Jaeger, Wuerzburg, Germany) were performed according to the European Respiratory Society (ERS) recommendations. ${ }^{8}$

Thereafter, all patients underwent a standardised 6 min walk test ${ }^{9}$ followed by a resting period of $60 \mathrm{~min}$. Finally an incremental exercise test was performed on an electromagnetically braked cycle ergometer (Ergoselect 200P; Ergoline, Bitz, Germany) with continuous measurements throughout rest ( $3 \mathrm{~min})$, unloaded pedalling $(3 \mathrm{~min})$ and incremental exercise (stepwise workload increments of 5 or $10 \mathrm{~W}$ every $2 \mathrm{~min}$ ) up to the limits of exercise tolerance. Patients were verbally encouraged before and during the test, to make a maximal effort. Standard criteria for termination of exercise were applied. ${ }^{10}$ All patients terminated exercise due to intolerable dyspnoea or exhaustion.

All waiting list patients used supplemental oxygen at rest and during the exercise tests. Cardiac frequency and arterial oxygen saturation measured by pulse oximetry were monitored continuously (SC 6000P, Siemens Medical Systems, Danvers, Massachusetts, USA). Blood pressure was measured by arm cuff every $2 \mathrm{~min}$. Borg ratings of dyspnoea and leg discomfort were assessed every $2 \mathrm{~min}$ at the end of each workload throughout exercise using a modified 1-10 Borg scale. ${ }^{11}$

\section{Opto-electronic plethysmography (OEP)}

During the exercise test, chest wall volumes were measured on a breath-by-breath basis by OEP (OEPsystem, BTS, Milano, Italy). OEP is based on six calibrated video cameras (placed three in front of the subject and three behind) which record the position of 89 reflective markers placed on the front and back over the chest wall from the clavicles to pubis. ${ }^{12} 13$ To prevent the arms from obscuring the markers, during cycling the patients grasped handles positioned laterally at the mid sternum level. From the three-dimensional coordinates of the markers obtained by stereo-photogrammetry, a specialised software based on the Gauss theorem provided continuous measurement of the volume of the total chest wall $\left(\mathrm{V}_{\mathrm{CW}}\right)$, split into rib cage $\left(\mathrm{V}_{\mathrm{RC}}\right)$ and abdomen $\left(\mathrm{V}_{\mathrm{AB}}\right)$, with $\mathrm{V}_{\mathrm{CW}}=\mathrm{V}_{\mathrm{RC}}+\mathrm{V}_{\mathrm{AB}}$

From chest wall volume measurements the complete set of ventilatory parameters including tidal volume $\left(\Delta \mathrm{V}_{\mathrm{CW}}\right)$, breathing frequency $\left(\mathrm{f}_{\mathrm{B}}\right)$, minute ventilation $\left(\mathrm{f}_{\mathrm{B}} \times \Delta \mathrm{V}_{\mathrm{CW}}\right)$, total respiratory cycle time $\left(\mathrm{T}_{\mathrm{TOT}}\right)$, inspiratory time $\left(\mathrm{T}_{\mathrm{I}}\right)$, mean inspiratory $\left(\Delta \mathrm{V}_{\mathrm{CW}} / \mathrm{T}_{\mathrm{I}}\right)$ flow rate and duty cycle $\left(\mathrm{T}_{\mathrm{I}} / \mathrm{T}_{\mathrm{TOT}}\right)$ was determined. End-expiratory (EE) and end-inspiratory (EI)

Table 1 Patient characteristics: anthropometric characteristics, blood gases, spirometric values, subdivision of lung volumes and exercise tolerance in patients with pulmonary fibrosis, cystic fibrosis, chronic obstructive pulmonary disease (COPD), healthy control subjects and transplanted patients

\begin{tabular}{|c|c|c|c|c|c|}
\hline & Pulmonary fibrosis & Cystic fibrosis & COPD & Healthy controls & Transplanted \\
\hline $\mathrm{n}$ & 9 & 9 & 21 & 10 & 16 \\
\hline Age (years) & $53.89 \pm 4.19$ & $32.89 \pm 3.05$ & $54.43 \pm 1.02$ & $52.20 \pm 2.624$ & $51.13 \pm 2.42$ \\
\hline Gender (M/F) & $4 / 5$ & $5 / 4$ & $8 / 13$ & $5 / 5$ & $11 / 5$ \\
\hline Height $(\mathrm{cm})$ & $170.22 \pm 3.87$ & $167.78 \pm 3.84$ & $167.93 \pm 1.99$ & $172.85 \pm 3.50$ & $174.56 \pm 2.65$ \\
\hline Weight (kg) & $64.21 \pm 4.95$ & $52.33 \pm 3.17$ & $58.51 \pm 2.29$ & $71.32 \pm 2.88$ & $75.48 \pm 3.92$ \\
\hline Body mass index $\left(\mathrm{kg} / \mathrm{m}^{2}\right)$ & $21.98 \pm 1.13$ & $18.33 \pm 0.57$ & $20.77 \pm 0.76$ & $23.92 \pm 0.82$ & $24.61 \pm 0.86$ \\
\hline $\mathrm{P}_{2}(\mathrm{kPa})$ & $8.19 \pm 0.81$ & $9.00 \pm 0.48$ & $9.05 \pm 0.35$ & - & $10.86 \pm 0.24$ \\
\hline $\mathrm{PcO}_{2}(\mathrm{kPa})$ & $4.94 \pm 0.37$ & $5.39 \pm 0.32$ & $5.59 \pm 0.23$ & - & $4.41 \pm 0.12$ \\
\hline FVC (litres) & $1.20 \pm 0.19$ & $1.21 \pm 0.13$ & $1.55 \pm 0.10$ & $4.04 \pm 0.40$ & $3.43 \pm 0.38$ \\
\hline FVC (\% predicted) & $31.71 \pm 2.55$ & $29.73 \pm 3.34$ & $45.66 \pm 2.50$ & $101.80 \pm 3.74$ & $79.73 \pm 5.85$ \\
\hline $\mathrm{FEV}_{1}$ (litres) & $1.17 \pm 0.19$ & $0.77 \pm 0.08$ & $0.58 \pm 0.03$ & $3.35 \pm 0.30$ & $2.79 \pm 0.31$ \\
\hline $\mathrm{FEV}_{1}(\%$ predicted $)$ & $37.88 \pm 3.56$ & $22.46 \pm 2.81$ & $20.77 \pm 1.15$ & $102.69 \pm 3.35$ & $79.40 \pm 6.46$ \\
\hline $\mathrm{FEV}_{1} / \mathrm{FVC}(\%)$ & $92.40 \pm 2.06$ & $65.40 \pm 4.10$ & $31.66 \pm 2.09$ & $80.84 \pm 1.74$ & $75.03 \pm 2.73$ \\
\hline TGV (litres) & $1.93 \pm 0.25$ & $4.48 \pm 0.48$ & $7.22 \pm 0.46$ & $3.55 \pm 0.35$ & $3.01 \pm 0.29$ \\
\hline TGV (\% predicted) & $61.67 \pm 7.46$ & $149.53 \pm 13.92$ & $241.07 \pm 12.73$ & $110.81 \pm 7.60$ & $91.60 \pm 8.92$ \\
\hline RV (litres) & $1.50 \pm 0.26$ & $4.16 \pm 0.49$ & $5.69 \pm 0.49$ & $2.44 \pm 0.16$ & $2.05 \pm 0.20$ \\
\hline RV (\% predicted) & $74.57 \pm 13.17$ & $253.74 \pm 24.89$ & $287.50 \pm 21.29$ & $121.36 \pm 6.76$ & $103.44 \pm 11.74$ \\
\hline TLC (litres) & $2.77 \pm 0.34$ & $5.65 \pm 0.54$ & $8.46 \pm 0.48$ & $6.63 \pm 0.57$ & $5.72 \pm 0.46$ \\
\hline TLC (\% predicted) & $45.84 \pm 3.96$ & $96.19 \pm 7.63$ & $149.54 \pm 6.28$ & $105.96 \pm 4.27$ & $87.33 \pm 5.92$ \\
\hline RV/TLC (\%) & $52.73 \pm 5.65$ & $72.41 \pm 3.05$ & $71.83 \pm 3.95$ & $37.46 \pm 1.39$ & $36.69 \pm 2.96$ \\
\hline Maximum workload (W) & $13.33 \pm 5.95$ & $25.56 \pm 6.03$ & $16.67 \pm 2.22$ & $159.00 \pm 17.35$ & $82.5 \pm 8.8$ \\
\hline Maximum workload (\% predicted $)^{7}$ & $7.6 \pm 3.1$ & $17.2 \pm 3.5$ & $13.8 \pm 2.2$ & $97.6 \pm 5.0$ & $46.8 \pm 5.3$ \\
\hline $6 \mathrm{MW}$ distance $(\mathrm{m})$ & $199.60 \pm 65.97(n=5)$ & $287.00 \pm 54.21(n=5)$ & $229.50 \pm 26.07(n=17)$ & - & - \\
\hline
\end{tabular}


volumes of the total chest wall and its compartments (rib cage and abdomen) were also measured by OEP as previously described elsewhere. ${ }^{13}$

\section{Statistical analysis}

Differences between the five groups (PF, CF, COPD, HC and T) were evaluated by parametric two-way analysis of variance (ANOVA), with subject group and condition (quiet breathing and maximal exercise workload) as the independent factors. In the cases where the data were not normally distributed, a nonparametric Kruskal-Wallis two-way ANOVA on ranks was applied. Posthoc tests were based on Holm-Sidak and Dunn methods, for parametric and non-parametric ANOVA tests, respectively. All data are expressed as mean \pm SE. Differences were regarded as significant with $p$ values $\leq 0.05$.

\section{RESULTS}

\section{Pulmonary function and exercise capacity}

As expected, we found significant differences between the groups regarding resting lung volumes and spirometry before lung transplantation (table 1). Exercise capacity was severely impaired in all three patient groups. In transplanted patients, exercise capacity was significantly higher than in PF, CF and COPD $(p<0.05)$ but lower than in healthy controls $(p<0.05)$.

\section{Breathing pattern}

Breathing pattern parameters at rest showed significant differences in the three patient groups and healthy controls regarding breathing frequency, timing, minute ventilation, duty cycle and mean inspiratory flow (see online supplement table 1S). Patients with PF had high breathing frequency compared with those with COPD and controls $(35.0 \pm 3.6 / \mathrm{min}, \mathrm{p}<0.001)$. Patients with CF also had high breathing frequency $(25.4 \pm 1.9 / \mathrm{min})$ compared with those with COPD and controls $(p<0.01$ with Dunn posthoc comparison, $\mathrm{Q}=4.159$ and $\mathrm{Q}=4.460$, respectively) but slightly reduced tidal volume $(0.55 \pm 0.04$ litres, non-significant (NS)), resulting in a high minute ventilation $(13.5 \pm 0.4 \mathrm{l} /$ min). In patients with PF and CF the duty cycle was either slightly higher or normal $(41.7 \pm 1.4 \%$ and $39.1 \pm 1.6 \%$, respectively). Breathing frequency and total respiratory cycle time $\left(\mathrm{T}_{\mathrm{TOT}}\right)$ were normal in patients with COPD, but the decreased $T_{I}$ and the increased expiratory time $\left(T_{E}\right)$ compared with controls determined a duty cycle lower than normal $(30.4 \pm 1.1 \%, \mathrm{p}<0.001)$.

At maximal exercise (table 2), patients with COPD maintained a duty cycle lower than that of all other groups (33.9 $\pm 1.1 / \mathrm{min}, \mathrm{p}<0.001)$. In patients with PF tidal volume did not increase compared with rest, and the increased minute ventilation compared with rest was achieved by a markedly increased breathing frequency $(49.4 \pm 6.1 / \mathrm{min}, \mathrm{p}<0.001)$. Patients with CF showed an intermediate pattern, with an increased tidal volume $(0.79 \pm 0.07$ litres, $\mathrm{p}<0.001)$ and a slightly increased breathing frequency $(33.9 \pm 2.3 / \mathrm{min})$.

Figures 1 and 2 summarise the results of breathing patterns at rest and during exercise in the different groups. Figure $1 \mathrm{~A}$ shows the relationship between tidal volume and breathing frequency at rest and during exercise in those with PF, CF and COPD and in healthy controls. Although the peak values of minute ventilation were much lower in subjects with COPD than in healthy controls, the progressive increase in minute ventilation of patients with COPD during exercise followed a pattern very similar to the normal one, with both tidal volume and breathing frequency increasing. In contrast, patients with PF and CF

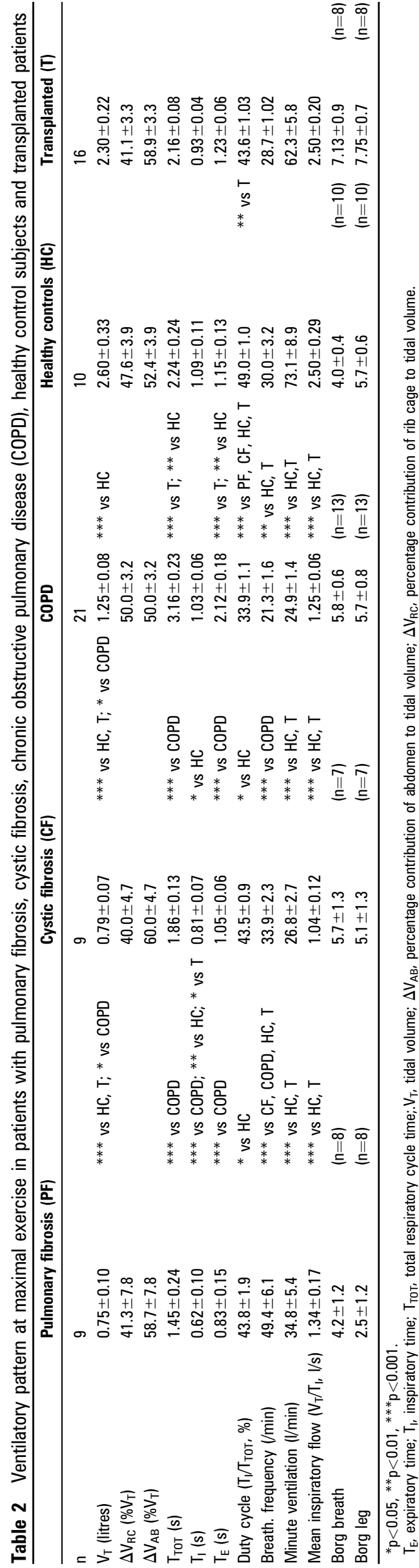


followed a completely different pattern, in which the increase in minute ventilation was achieved almost exclusively by increasing breathing frequency (rapid and shallow breathing). Figure $1 \mathrm{~B}$ shows that transplanted patients had a frequency-tidal volume pattern almost identical to that of healthy subjects.

Minute ventilation can additionally be considered as the product of mean inspiratory flow $\left(\mathrm{V}_{\mathrm{T}} / \mathrm{T}_{\mathrm{I}}\right)$ and duty cycle $\left(\mathrm{T}_{\mathrm{I}} /\right.$ $\mathrm{T}_{\text {TOT }}$ ). In figure $2 \mathrm{~A}$ these two variables are plotted against each other in patients with PF, CF and COPD and in healthy controls. Patients with PF and CF showed a pattern similar to that of controls. In contrast, patients with COPD had a distinct pattern, with a significantly lower duty cycle and increased mean inspiratory flow at any minute ventilation. Figure $2 \mathrm{~B}$ shows that after transplantation, all patients restored a duty cycle-mean inspiratory flow pattern similar to that of healthy controls, particularly at the lower level of exercise.
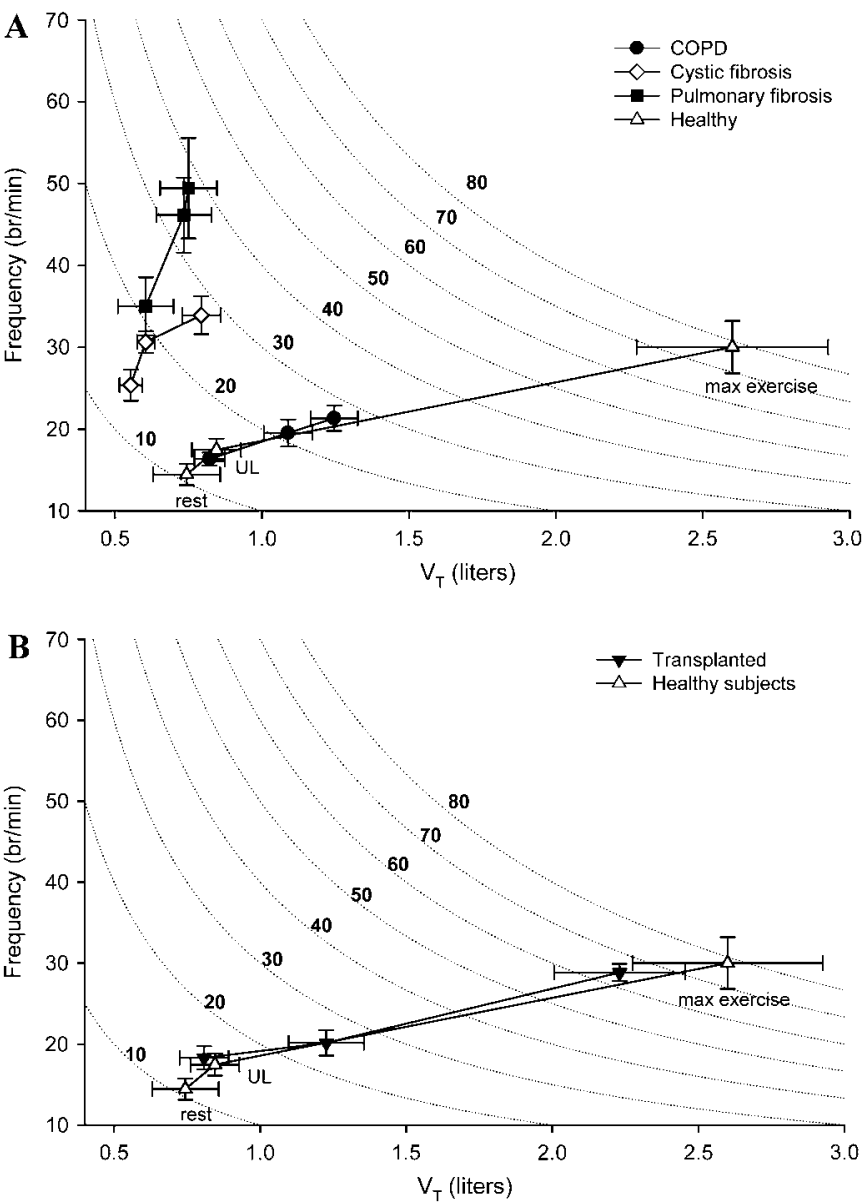

Figure 1 (A) Relationship between the mean values $\pm S E$ of tidal volume $\left(\mathrm{V}_{\mathrm{T}}, x\right.$-axis) and respiratory frequency ( $y$-axis) at rest, unloaded (UL) and during maximal exercise in the groups of patients with pulmonary fibrosis (filled squares), cystic fibrosis (open diamonds) and chronic obstructive pulmonary disease (COPD; filled circles) and in healthy control subjects (open triangles). Dotted lines represent isopleths of different levels of minute ventilation from 10 to $80 \mathrm{l} / \mathrm{min}$, according to the relationship $\dot{V}_{E}=V_{T} \times f_{B}$. (B) Relationship between the mean values $\pm S E$ of $V_{T}$ ( $x$-axis) and respiratory frequency ( $y$-axis) at rest, $U L$ and during maximal exercise in the groups of transplanted patients (filled inverted triangles) and healthy control subjects (open triangles). Dotted lines represent isopleths of different levels of minute ventilation from 10 to $80 \mathrm{l} / \mathrm{min}$, according to the relationship $\dot{\mathrm{V}}_{\mathrm{E}}=\mathrm{V}_{\mathrm{T}} \times \mathrm{f}_{\mathrm{B}}\left(\dot{\mathrm{V}}_{\mathrm{E}}=\right.$ minute ventilation; $f_{B}=$ breathing frequency).
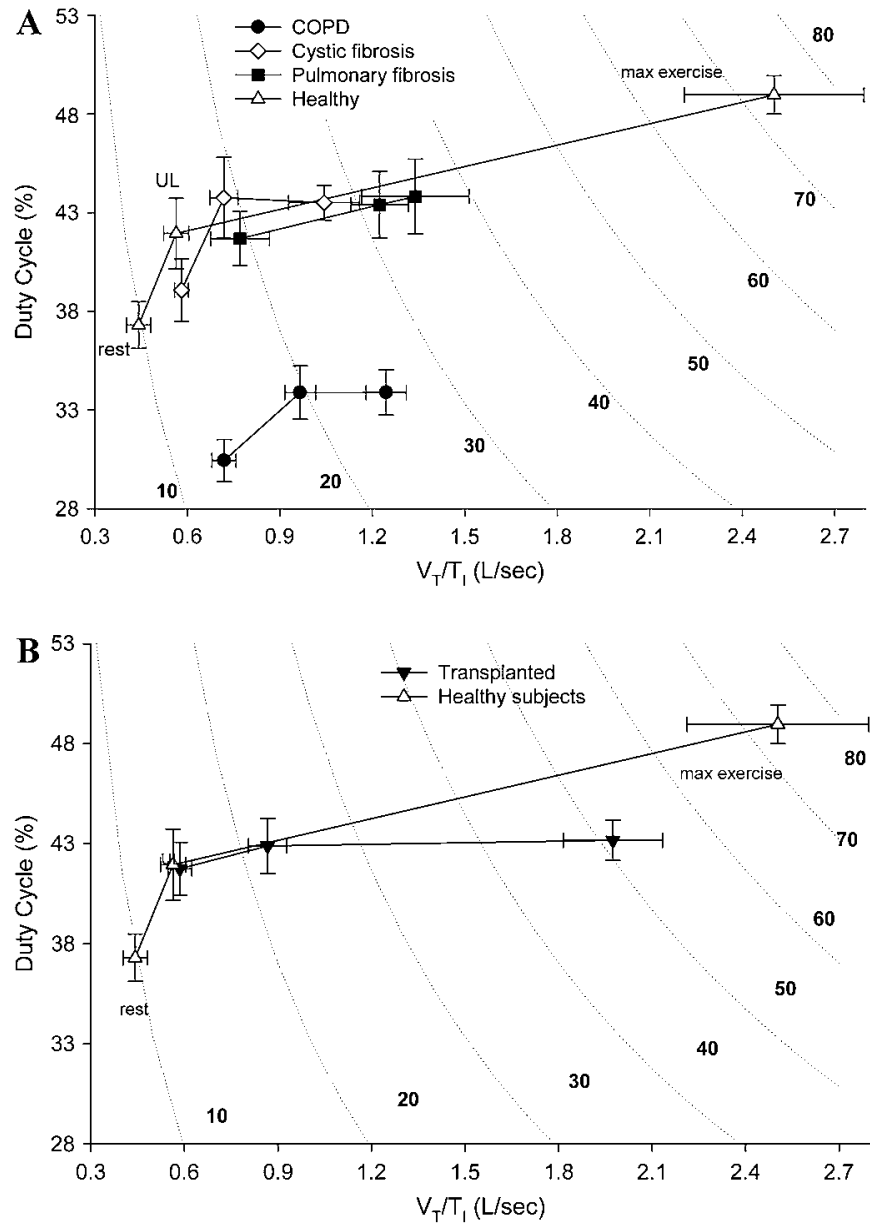

Figure 2 (A) Relationship between the mean values $\pm S E$ of mean inspiratory flow $\left(\mathrm{V}_{\mathrm{T}} / \mathrm{T}_{1}, x\right.$-axis) and duty cycle $\left(\mathrm{T}_{/} / \mathrm{T}_{\mathrm{TOT}}, y\right.$-axis $)$ at rest unloaded (UL) and during maximal exercise in the groups of patients with pulmonary fibrosis (filled squares), cystic fibrosis (open diamonds) and chronic obstructive pulmonary disease (COPD; filled circles) and in healthy control subjects (open triangles). Dotted lines represent isopleths of different levels of minute ventilation from 10 to $80 \mathrm{l} / \mathrm{min}$, according to the relationship $\dot{V}_{E}=60 \times\left(\frac{V_{T}}{T_{1}}\right) \times\left(\frac{T_{1}}{T_{T O T}}\right)$. (B) Relationship between the mean values $\pm S E$ of mean $V_{T} / T_{1}$ ( $x$-axis) and $T_{1} / T_{\text {TOT }}$ ( $y$-axis) at rest, UL and during maximal exercise in the groups of transplanted patients (filled inverted triangle) and healthy control subjects (open triangle). Dotted lines represent isopleths of different levels of minute ventilation from 10 to $80 \mathrm{l} / \mathrm{min}$, according to the relationship $\dot{\mathrm{V}}_{\mathrm{E}}=60 \times\left(\frac{\mathrm{V}_{\mathrm{T}}}{\mathrm{T}_{\mathrm{I}}}\right) \times\left(\frac{\mathrm{T}_{\mathrm{I}}}{\mathrm{T}_{\mathrm{T} \text { T }}}\right) .\left(\dot{\mathrm{V}}_{\mathrm{E}}=\right.$ minute ventilation; $V_{T}=$ tidal volume; $T_{1}=$ inspiratory time; $\mathrm{T}_{\mathrm{TOT}}=$ total respiratory cycle time).

\section{Chest wall volumes}

The percentage contributions of the rib cage and abdominal compartment to tidal volume were similar in all the groups, while the EE and EI total and compartmental chest wall volumes showed differences. Figure 3 illustrates the EE and EI values of the total chest wall volume at unloaded and maximal exercise compared with rest. In these plots, the difference between EI and EE points at any given exercise workload represents tidal volume. In healthy controls, the progressive increase in tidal volume was due to an increase in EI $(p<0.001$, unloaded and maximal exercise compared with rest) and a decrease in $\mathrm{EE}$ ( $p=0.042$, maximal exercise compared with rest). In patients with $\mathrm{PF}$ there was almost no change in $\mathrm{EI}$ and $\mathrm{EE}$, resulting in an unchanged tidal volume. In patients with CF the tidal volume increased slightly, with a progressive increase in both EE and EI ( $p=0.021$ and $p=0.019$, respectively, maximal 
exercise compared with rest). In patients with COPD, both EE $(p=0.069$ and $p=0.042$, unloaded and maximal exercise compared with rest, respectively) and EI ( $p=0.028$ unloaded exercise vs rest; $p=0.005$ unloaded vs maximal exercise; $p<0.001$ maximal exercise vs rest) increased or tended to increase during exercise. The increase of EI, however, was higher and consequently tidal volume increased significantly during exercise $(p=0.067$ unloaded exercise vs rest; $p=0.004$ maximal exercise vs rest). Only 3 out of 21 patients with COPD decreased EE volume at maximal exercise. The comparison between the overall volume data in the three patient groups before lung transplantation revealed that EI and tidal volumes were higher in COPD than in PF and CF $(p<0.001)$. In the transplanted patients, EE, EI and tidal volumes were similar to those of healthy controls at any given level of workload.

Figure 4 illustrates how EE and EI volume variations are divided into the rib cage (top panels) and abdominal (bottom panels) compartments. In healthy subjects, the increase of EI volume of the total chest wall compared with rest was due to both the rib cage and the abdomen $(p<0.001)$, while the decrease of EE volume was totally achieved in the abdomen $(p<0.001)$. In patients with PF, no volume changes occurred in either compartment. In patients with CF, the increase of total EE and EI chest wall volumes at maximal exercise compared with rest was totally due to the rib cage compartment $(p=0.003$ and $p=0.005$, respectively). The increase in tidal volume was mainly due to the abdomen. In patients with COPD, the increase in total EI chest wall volume compared with rest was due to both the rib cage $(p<0.001)$ and the abdomen $(p=0.017)$. On the other hand, the increase of total EE chest wall volume compared with rest was only due to the rib cage $(p<0.001)$. The increase in tidal volume compared with rest was due to both the rib cage $(p=0.035)$ and abdomen $(p=0.016)$. In the transplanted patients, $\mathrm{EE}, \mathrm{EI}$ and tidal volumes of the rib cage and the abdomen were similar to those of healthy controls.

\section{DISCUSSION}

This study represents the first systematic comparison of breathing pattern and chest wall volume changes during quiet breathing and exercise between patients with PF, CF or COPD, transplanted patients and healthy subjects. The ventilatory pattern of these diseases has been described so far only separately, ${ }^{8}{ }^{14-19}$ and a comprehensive comparison between the most advanced disease stages and healthy controls or transplanted patients has not been available.

The main finding of this study is that each disease results in a different breathing pattern during chronic respiratory failure. The reduced duty cycle represents the main feature of alteration in COPD, while the increased breathing frequency and decreased tidal volume is the characteristic distinguishing patients with PF or CF.

\section{Patients with COPD}

In patients with COPD expiratory flow limitation determines longer expiratory time, inducing significantly lower values of the duty cycle and a limited minute ventilation both at rest and during exercise ( $\sim 30 \%$ compared with age-matched healthy subjects). In a recent paper, ${ }^{20}$ the duty cycle in patients with COPD with less advanced disease was also decreased compared with normal, being, however, considerably higher than in our waiting list patients. These findings suggest that the duty cycle is inversely correlated with the severity of the disease. Previous data have also shown that this parameter can be used in patients with COPD to predict the need for mechanical ventilation in acute exacerbations. ${ }^{21}$ Another significant abnormality in the pattern of breathing of patients with COPD is the high inspiratory flow rate resulting from the low duty cycle and high minute ventilation. A high inspiratory flow rate in the face of airways obstruction indicates a greater than normal inspiratory pressure drop with an abnormally high central inspiratory drive. This is probably a major cause of dyspnoea and thus exercise limitation in COPD.

Our data also show that patients with COPD exhibit a preserved tidal volume at rest. During exercise it increases both in the rib cage and in the abdomen, but it is limited by dynamic hyperinflation of the chest wall. This is located in the rib cage. $^{1322}$ In our waiting list patients, 3 out of 21 showed an euvolumic pattern ${ }^{13}$ - that is, a decrease of EE chest wall volume during exercise. Interestingly, these three patients were the ones showing the best predicted forced expiratory volume in $1 \mathrm{~s}$ $\left(\mathrm{FEV}_{1}\right)$ in the overall group of patients with COPD, suggesting that dynamic hyperinflation occurs mainly in the most severe patients. ${ }^{13}$
Figure 3 End-inspiratory (open symbols) and end-expiratory (filled symbols) total chest wall volume variations at rest, unloaded (UL) and during maximal exercise in the groups of patients with pulmonary fibrosis (left panel), cystic fibrosis (left middle panel) and chronic obstructive pulmonary disease (COPD; right middle panel). Healthy control subjects and transplanted patients are shown in the right panel. Each point represents the mean $\pm S E$. All values are referred to the end-expiratory chest wall volume at rest. ${ }^{* *}{ }^{* * *}, \mathrm{p}<0.01, \mathrm{p}<0.001$ (vs healthy); \#\#, \#\#\#, p <0.01, p $<0.001$ (vs transplanted patients); $\hat{\wedge}$, ^ ^ ^, $\mathrm{p}<0.01, \mathrm{p}<0.001$ (vs COPD); ${ }^{\circ}, 000, \mathrm{p}<0.01, \mathrm{p}<0.001$ (vs rest); $\S$, $\S \S \S: \mathrm{p}<0.05, \mathrm{p}<0.001$ (vs UL).

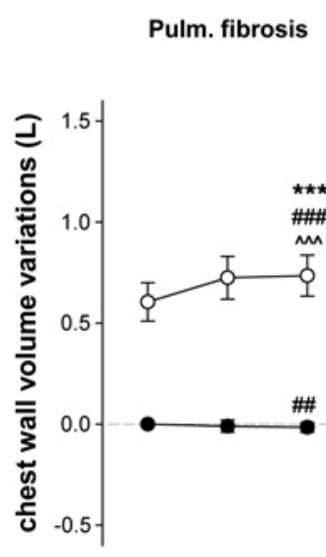

rest UL Wmax

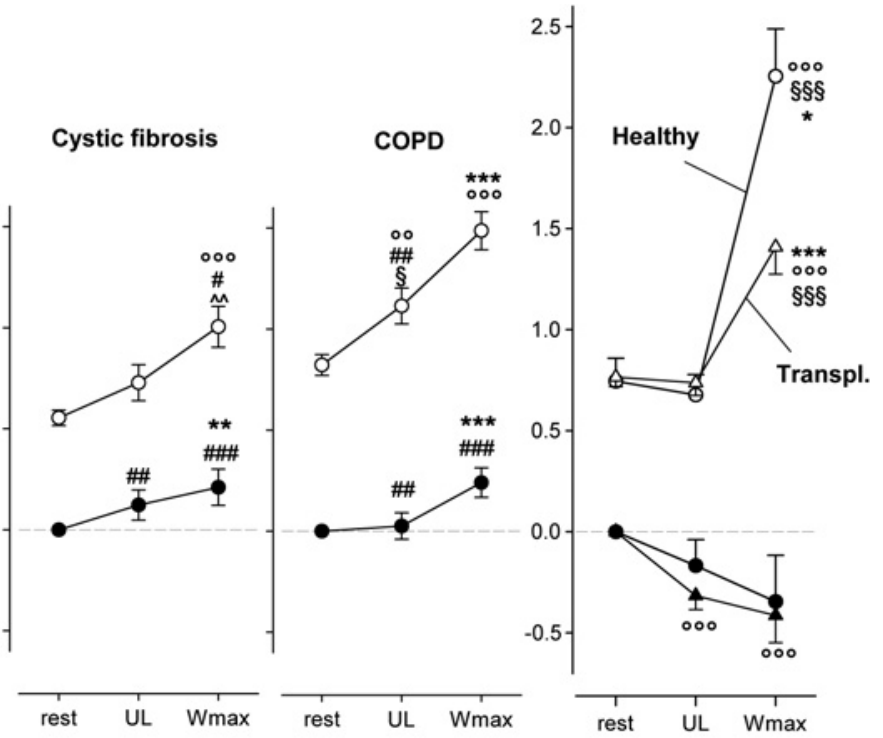

Workload 
Figure 4 Top panels: end-inspiratory (open symbols) and end-expiratory (filled symbols) volume variations of the rib cage at rest, unloaded (UL) and during maximal exercise in the groups of patients with pulmonary fibrosis (left panel), cystic fibrosis (left middle panel) and chronic obstructive pulmonary disease (COPD; right middle panel). Healthy control subjects and transplanted patients are shown in the right panel. Each point represents mean $\pm S E$. All values are referred to end-expiratory rib cage volume at rest. Bottom panels: end-inspiratory (open symbols) and end-expiratory (filled symbols) volume variations of the abdomen at rest, UL and during maximal exercise in the groups of patients with pulmonary fibrosis (left panel), cystic fibrosis (left-middle panel) and COPD (right-middle panel). Healthy control subjects and transplanted patients are shown in the right panel. Each point represents the mean \pm SE. All values are referred to end-expiratory abdominal volume at rest. ${ }^{* *},{ }^{* *}, \mathrm{p}<0.01, \mathrm{p}<0.001$ (vs healthy); \#\#, \#\#\#, $\mathrm{p}<0.01, \mathrm{p}<0.001$ (vs transplanted patients); $\wedge, \mathrm{p}<0.05$ (vs COPD); ${ }^{\circ}$ $\circ,, p>0.05, \mathrm{p}<0.01, \mathrm{p}<0.001$ (vs rest); $\S \S, \S \S \S, \mathrm{p}<0.01, \mathrm{p}<0.001$ (vs UL).

Frequency/tidal volume variations during both quiet breathing and exercise resembled those found in healthy subjects and are in agreement with data obtained by respiratory inductive plethysmography in patients with moderate COPD. ${ }^{23}$ It has been shown that in patients with COPD, hypercapnia and rapid shallow breathing are very closely related during acute respiratory failure. ${ }^{24-26}$ In our results obtained in patients with COPD this pattern did not occur, even at very severe stages of the disease. The reason for this might be that our patients awaiting transplantation were highly selected, receiving carefully monitored optimal treatment, and therefore might not be representative of the entire population of patients with severe disease.

\section{Patients with PF}

Patients with PF exhibit at rest a rapid and shallow breathing with low values of tidal volume and very high breathing frequencies. Possible mechanisms involved in this pattern of breathing include increased lung elastance, perceived as increasing load by mechanoreceptors, and stimulation of intrapulmonary receptors, such as rapidly adapting and J receptors. ${ }^{27}$ Rib cage tidal volume is decreased both at rest and during exercise, probably due to the increased rigidity of the lung and consequently to the reduced expansion of the rib cage compartment apposed to the lung. Total minute ventilation is higher than normal, permitting an increased alveolar ventilation to overcome disease-specific gas exchange alteration. Additionally, a high dead space occurring with a low tidal volume might contribute to this altered breathing pattern. Excessive dead space ventilation is common in interstitial lung diseases, leading to a higher minute ventilation and ventilatory work during exercise. ${ }^{15}$ At maximal exercise the doubled minute ventilation is totally due to the increased breathing frequency since tidal volume and its subdivision remain completely unchanged. The associated increase in the metabolic energy requirement of respiratory muscles may exceed $50 \%$ of available total body oxygen delivery and result in insufficient energy delivery to nonrespiratory muscles that sustain locomotion. ${ }^{28}$ This also occurs in COPD and presumably leads to premature competition between locomotor and respiratory muscles for available energy supplies, which is undoubtedly a major contributor to exercise limitation. ${ }^{29}$

\section{Patients with CF}

Patient with CF show a reduced tidal volume at rest. Breathing frequency is significantly higher than in normal subjects but lower than in patients with $\mathrm{PF}$, resulting in high minute ventilation that, associated with hypercapnia, indicates a grossly abnormal alveolar dead space. During exercise, these patients are able to increase tidal volume slightly $(p<0.01)$ mainly by increasing abdominal displacement. These data are in agreement with those of Keochkerian et al, ${ }^{30}$ who reported that during exercise, children with CF, even those not suffering from advanced disease, showed signs of rapid and shallow breathing and an increase in the ventilatory response. This was essentially due to an increase in the mean inspiratory flow, which in turn suggests an expiratory flow limitation. ${ }^{31}$ Indeed, our patients with CF have dynamic hyperinflation of the chest wall. Likewise, in patients with COPD, the increase in EE chest wall volume is located in the rib cage compartment. Our results show that patients with CF are restricted at end-inspiration but dynamically hyperinflate with exercise, while patients with PF are restricted at both end-expiration and end-inspiration.

The situation in patients with CF depends on the severity of the disease. With our data one can speculate that during the progression of the disease in $\mathrm{CF}$, frequency/tidal volume ratios gradually tend from normal values to those observed in patients with PF. These functional changes might reflect the structural transition from the progressive airflow obstruction, caused by mucus plugging and inflammation within the bronchial walls, to the destruction of the lung parenchyma secondary to bronchiectasis. ${ }^{32}$ Our data reveal that in several aspects patients with CF have an intermediate pattern between that of patients with COPD and that of patients with PF, which is somewhat closer to $\mathrm{PF}$ than COPD in the most advanced states of disease.

\section{Transplanted patients}

Our data show that lung transplantation restores a normal breathing pattern regardless of the underlying disease. The maximal exercise capacity, however, is significantly lower than 
that of healthy subjects. ${ }^{33}$ Several factors contributing to the reduced exercise performance have been discussed, including cardiovascular and muscular deconditioning due to inactivity ${ }^{34}$ and intake of immunosuppressive agents. ${ }^{35}$ Additionally, it has been reported that patients transplanted for diseases that produce chronic hyperinflation, such as CF and emphysema, may have preoperative structural changes in rib cage shape that persist in part after transplantation and lead to persistent increases in functional residual capacity and residual volume. ${ }^{36}$ These alterations, when still present after transplantation, might contribute to decreased exercise tolerance.

It was not our purpose to analyse in detail differences between the three patient groups after lung transplantation or differences between single and double lung transplantation. Nevertheless, no significant differences were found in the three diseases after transplantation either in the breathing pattern or in chest wall behaviour at rest and during exercise. Retrospective analysis of pretransplant pulmonary function data of the transplanted patients (split into PF, CF and COPD) showed that their characteristics were almost identical to those of the patients with PF, CF and COPD (see online supplement).

Besides the selection of the patients (see above), the main limitation of our study is that the patients after transplantation are not identical to those before transplantation. In the online data supplement, however, it is shown that the lung function of these groups is matched. Another limitation is that exercise data are presented only during the unloaded cycling and maximal workload. This was due to the very limited exercise capacity of our patients, since half of them could only perform one level of exercise.

In conclusion, we showed that there are three distinct patterns of chronic respiratory failure in patients with $\mathrm{PF}, \mathrm{CF}$ and COPD adopted by the ventilatory pump to cope with the underlying lung disease. Our data provide new evidence as to why patients with PF and, to a lesser extent, with CF are prone to respiratory failure much earlier than patients with COPD. The ventilatory pump of patients with PF and CF lacks any reserve, since coping with increasing elastic load leads to high breathing frequency. After lung transplantation, these chronic adaptations of the ventilatory pattern to advanced lung diseases are reversible and may indicate that the main contributing factor is the lung itself rather than systemic effects of the disease. We believe that our results will be helpful for optimising pulmonary rehabilitation before and after lung transplantation as well as weaning from mechanical ventilation after acute exacerbations.

Funding The European Community CARED FP5 project (QLG5-CT-2002-0893). Competing interests None.

Ethics approval This study was conducted with the approval of the ethics committee of the Ärztekammer des Saarlandes, Germany.

Provenance and peer review Not commissioned; externally peer reviewed.

\section{REFERENCES}

1. Glanville AR, Estenne M. Indications, patient selection and timing of referral for lung transplantation. Eur Respir J 2003;22:845-52.

2. Titman A, Rogers CA, Bonser RS, et al. Disease-specific survival benefit of lung transplantation in adults: a national cohort study. Am J Transplant 2009;9:1640-9.

3. Orens JB, Estenne M, Arcasoy S, et al. International guidelines for the selection of lung transplant candidates: 2006 update-a consensus report from the Pulmonary Scientific Council of the International Society for Heart and Lung Transplantation. J Heart Lung Transplant 2006;25:745-55.

4. O'Sullivan BP, Freedman SD. Cystic fibrosis. Lancet 2009;373:1891-90.
5. Gallagher CG, Younes M. Breathing pattern during and after maximal exercise in patients with chronic obstructive lung disease, interstitial lung disease, and cardiac disease, and in normal subjects. Am Rev Respir Dis 1986;133:581-6.

6. Otis AB, Fenn WO, Rahn H. Mechanics of breathing in man. J Appl Physiol 1950;2:592-607

7. Jones NL, Makrides L, Hitchcock C, et al. Normal standards for an incremental progressive cycle ergometer test. Am Rev Respir Dis 1985;131:700-8.

8. Miller MR, Crapo R, Hankinson J, et al. General considerations for lung function testing. Eur Respir J 2005;26:153-61.

9. ATS Committee on Proficiency Standards for Clinical Pulmonary Function Laboratories. ATS statement: guidelines for the six-minute walk test. Am J Respir Crit Care Med 2002:166:111-17.

10. American Thoracic Society. American college of chest physicians. ATS/ACCP statement on cardiopulmonary exercise testing. Am J Respir Crit Care Med 2003:167:211-77.

11. Ward ME, Eidelman D, Stubbing DG, et al. Respiratory sensation and pattern of respiratory muscle activation during diaphragm fatigue. J Appl Physiol 1988;65:2181-9

12. Cala SJ, Kenyon CM, Ferrigno G, et al. Chest wall and lung volume estimation by optical reflectance motion analysis. J App/ Physiol. 1996;81:2680-9.

13. Aliverti A, Stevenson N, Dellacà RL, et al. Regional chest wall volumes during exercise in chronic obstructive pulmonary disease. Thorax 2004;59:210-16.

14. Brack T, Jubran A, Tobin MJ. Dyspnea and decreased variability of breathing in patients with restrictive lung disease. Am J Respir Crit Care Med 2002;165:1260-4.

15. Burdon JG, Killian KJ, Jones NL. Pattern of breathing during exercise in patients with interstitial lung disease. Thorax 1983;38:778-84.

16. Fernandes $\mathbf{M}$, Cukier $\mathrm{A}$, Ambrosino $\mathrm{N}$, et al. Respiratory pattern, thoracoabdominal motion and ventilation in chronic airway obstruction. Monaldi Arch Chest Dis 2007;67:209-16.

17. Gaultier C, Perret L, Boule M, et al. Control of breathing in children with interstitia lung disease. Pediatr Res 1982;16:779-83.

18. Nield M, Arora A, Dracup K, et al. Comparison of breathing patterns during exercise in patients with obstructive and restrictive ventilatory abnormalities. J Rehabil Res Dev 2003:40:407-14.

19. Renzi G, Milic-Emili J, Grassino AE. The pattern of breathing in diffuse lung fibrosis Bull Eur Physiopathol Respir 1982;18:461-72.

20. Aliverti A, Quaranta M, Chakrabarti B, et al. Paradoxical movement of the lower ribcage at rest and during exercise in COPD patients. Eur Respir $J$ 2009;33:49-60.

21. Vitacca M, Clini E, Porta R, et al. Acute exacerbations in patients with COPD: predictors of need for mechanical ventilation. Eur Respir J 1996;9:1487-93.

22. Vogiatzis I, Georgiadou 0 , Golemati S, et al. Patterns of dynamic hyperinflation during exercise and recovery in patients with severe chronic obstructive pulmonary disease. Thorax 2005;60:723-9.

23. Alves GS, Britto RR, Campos FC, et al. Breathing pattern and thoracoabdominal motion during exercise in chronic obstructive pulmonary disease. Braz J Med Biol Res 2008;41:945-50.

24. Tobin MJ, Laghi F, Brochard L. Role of the respiratory muscles in acute respiratory failure of COPD: lessons from weaning failure. J Appl Physiol 2009;107:962-70.

25. Calverley PM. Respiratory failure in chronic obstructive pulmonary disease Eur Respir J Supp/ 2003;47:26s-30.

26. Javaheri S, Blum J, Kazemi H. Pattern of breathing and carbon dioxide retention in chronic obstructive lung disease. Am J Med 1981;71:228-34.

27. Javaheri S, Sicilian L. Lung function, breathing pattern, and gas exchange in interstitial lung disease. Thorax 1992;47:93-7.

28. Hsia CC. Cardiopulmonary limitations to exercise in restrictive lung disease. Med Sci Sports Exerc 1999;31(Suppl 1):S28-32.

29. Aliverti A, Macklem PT. The major limitation to exercise performance in COPD is inadequate energy supply to the respiratory and locomotor muscles. J Appl Physio 2008; 105:749-51.

30. Keochkerian D, Chlif M, Delanaud S, et al. Breathing pattern adopted by children with cystic fibrosis with mild to moderate pulmonary impairment during exercise. Respiration 2008;75:170-7.

31. Goetghebeur D, Sarni D, Grossi Y, et al. Tidal expiratory flow limitation and chronic dyspnoea in patients with cystic fibrosis. Eur Respir J 2002;19:492-8.

32. Hart N, Polkey MI, Clément A, et al. Changes in pulmonary mechanics with increasing disease severity in children and young adults with cystic fibrosis Am J Respir Crit Care Med 2002;166:61-6.

33. Schwaiblmair M, Reichenspurner $\mathrm{H}$, Müller $\mathrm{C}$, et al. Cardiopulmonary exercise testing before and after lung and heart-lung transplantation. Am J Respir Crit Care Med 1999;159:1277-83.

34. Williams T, Patterson G, McClean P, et al. Maximal exercise testing in single and double lung transplant recipients. Am Rev Respir Dis 1992;145:101-5.

35. Mercier JG, Hokanson JF, Brooks GA. Effects of cyclosporine A on skeletal muscle mitochondrial respiration and endurance time in rats. Am J Respir Crit Care Med 1995;151:1532-6.

36. Estenne M. Effect of lung transplant and volume reduction surgery on respiratory muscle function. J Appl Physiol 2009;107:977-86. 